À l'intersection des discours de spécialité :

hétérogénéité et unité

\title{
Zoltán Dörnyei, The Psychology of Second Language Acquisition
}

Oxford: Oxford University Press, 2009

\section{Cédric Brudermann}

\section{OpenEdition \\ Journals}

Édition électronique

URL : http://journals.openedition.org/asp/1805

DOI : 10.4000/asp.1805

ISBN : 978-2-8218-0414-2

ISSN : 2108-6354

Éditeur

Groupe d'étude et de recherche en anglais de spécialité

\section{Édition imprimée}

Date de publication : 30 novembre 2010

Pagination : 145-146

ISSN : 1246-8185

\section{Référence électronique}

Cédric Brudermann, «Zoltán Dörnyei, The Psychology of Second Language Acquisition », ASp [En ligne], 58 | 2010, mis en ligne le 30 novembre 2010, consulté le 07 novembre 2020. URL : http:// journals.openedition.org/asp/1805; DOI : https://doi.org/10.4000/asp.1805

Ce document a été généré automatiquement le 7 novembre 2020.

Tous droits réservés 


\section{Zoltán Dörnyei, The Psychology of Second Language Acquisition}

Oxford: Oxford University Press, 2009

Cédric Brudermann

\section{RÉFÉRENCE}

Dörnyei Zoltán. 2009. The Psychology of Second Language Acquisition. Oxford: Oxford University Press, 339 p. ISBN 978-0-19-442197-3. 
1 L'auteur de The Psychology of Second Language Acquisition, Zoltán Dörnyei ${ }^{1}$, est professeur de psycholinguistique à l'Université de Nottingham en GrandeBretagne. Il a publié de nombreux ouvrages portant notamment sur l'impact de la motivation, des différences individuelles et des croyances des apprenants concernant le processus d'acquisition des langues étrangères (second language acquisition).

2 The Psychology of Second Language Acquisition correspond à une synthèse des apports théoriques et méthodologiques les plus récents en "psycho-ALE » (acquisition des langues étrangères), un champ de recherche hybride dont la finalité est de dégager des connaissances sur les mécanismes psychologiques

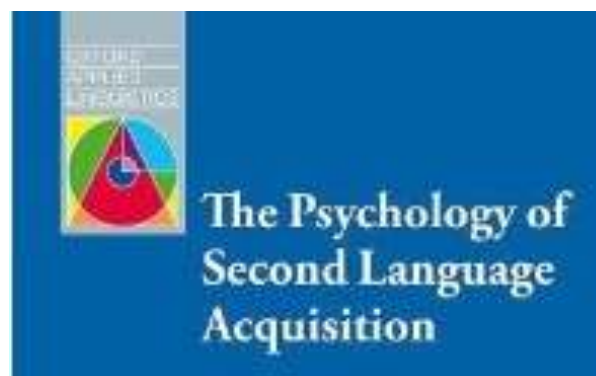

Zoltán Dörnyci impliqués dans le processus d'appropriation des langues, qu'elles soient maternelles ou secondes (désormais L2).

Ce champ découle de la convergence de la linguistique et de la psychologie, deux disciplines pour lesquelles l'objet « langue » revêt un intérêt particulier. Selon l'auteur, le croisement de ces deux disciplines aurait conduit les chercheurs en ALE, au sens large, à appréhender ce processus selon de nouvelles perspectives. Cette tendance s'est traduite par l'émergence d'une multitude de nouvelles disciplines qui, telles la neurolinguistique ou les neurosciences cognitives, font l'objet d'une présentation dans le premier chapitre de l'ouvrage.

4 Pour autant, les outils et la terminologie auxquels ces champs de recherche font appel peuvent constituer des freins pour des scientifiques qui, tels les didacticiens ou les linguistes, ne disposent pas nécessairement de formation universitaire antérieure en psychologie et/ou en sciences cognitives. L'objectif du deuxième chapitre est de pallier ce manque en faisant l'état de l'art des connaissances liées au fonctionnement du cerveau humain et en introduisant diverses méthodes (électrophysiologique, hémodynamique, imagerie à résonance magnétique, etc.) pour l'analyser.

5 Le chapitre trois est davantage centré sur la question du fonctionnement langagier au sein du cerveau. Pour en rendre compte, Z. Dörnyei revient tout d'abord sur les positionnements théoriques des écoles symbolique et connexionniste. Il introduit ensuite des paradigmes plus récents qui, comme l'émergentisme ou la théorie des modèles dynamiques, constituent des alternatives pour la compréhension du processus d'acquisition de la compétence langagière. Enfin, au chapitre quatre, les mécanismes cognitifs inhérents au fonctionnement de la mémoire et à la gestion des connaissances langagières (implicites, explicites et incidentes) sont abordés.

6 Le cinquième chapitre a trait à l'impact des différences individuelles (possibilités cognitives offertes par la mémoire de travail, l'aptitude langagière, les traits de personnalité...) et des variables sociales et affectives (émotions, motivation, styles 
d'apprentissage...) propres à chaque individu sur le processus cognitif de l'appropriation linguistique en L2. Au chapitre suivant, Z. Dörnyei relance le débat sur l'incidence de l'âge sur le niveau de compétence potentiellement accessible en L2, ainsi que sur l'existence de ladite "période critique", au-delà de laquelle les différentes aires du cerveau perdraient de leur plasticité et ne pourraient traiter les données relatives aux L2 qu'au travers d'un effort conscient et laborieux (Lenneberg 1967).

Enfin, le dernier chapitre pose la question de l'interprétation des travaux de la recherche sur l'acquisition des langues étrangères en didactique des langues et des cultures, notamment pour faire émerger des outils et des méthodologies susceptibles de conduire les apprenants à progresser dans leur apprentissage des L2. Pour ce faire, après un récapitulatif de diverses méthodes d'enseignement/apprentissage des L2 utilisées au cours du siècle précédent (grammaire-traduction, méthode audiolinguale, approche communicative), l'auteur propose une réflexion sur les apports de l'approche par les tâches (task-based language teaching and learning) et de l'enseignement de la grammaire orienté vers la forme (form-focused instruction).

8 Cet ouvrage, en offrant un état de la recherche sur divers aspects propres au champ de la psycho-ALE, constitue une étude incontournable pour quiconque souhaite s'initier ou se mettre à jour dans un domaine qui suscite actuellement un intérêt certain, ainsi qu'en témoignent les nombreuses publications récentes qui figurent dans la bibliographie.

9 En s'inspirant des apports de disciplines aussi différentes que la linguistique, la psychologie et la didactique des langues et des cultures sur le champ de l'acquisition des langues étrangères, Z. Dörnyei propose ici une interface pour appréhender et se construire un regard critique sur ce qui se joue aujourd'hui dans un domaine encore nouveau mais qui, selon les propos de l'auteur, sera amené à connaître de profonds bouleversements. En tant que tel, l'ouvrage permet de préparer le terrain pour les débats, méthodologies et paradigmes théoriques à venir.

\section{BIBLIOGRAPHIE}

Lenneberg, Eric. 1967. Biological Foundations of Language. New York : John Wiley \& Sons.

\section{NOTES}

1. Site personnel < http://www.nottingham.ac.uk/ aezweb/research/cral/doku.php? id=people:zoltan> 


\section{AUTEURS}

\section{CÉDRIC BRUDERMANN}

Université Paris 6 